\title{
Application of hairless mouse strain to bioluminescence imaging of Arc expression in mouse brain
}

Hironori Izumi ${ }^{1}$, Tetsuya Ishimoto' ${ }^{1}$, Hiroshi Yamamoto ${ }^{2}$ and Hisashi Mori ${ }^{*}$

\begin{abstract}
Background: Bioluminescence imaging (BLI) is a powerful technique for monitoring the temporal and spatial dynamics of gene expression in the mouse brain. However, the black fur, skin pigmentation and hair regrowth after depilation of mouse interfere with BLI during developmental and daily examination. The aim of this study was to extend the application of Arc-Luc transgenic ( $\mathrm{Tg}$ ) mice to the BLI of neuronal activity in the mouse brain by introducing the hairless ( $\mathrm{HL}$ ) gene and to examine Arc-Luc expression at various developmental stages without interference from black fur, skin pigmentation, and hair regrowth.

Results: The Arc-Luc Tg HL mice were established by crossing the Tg C57BL/6 mouse strain with the HL mouse strain. Under physiological and pathological conditions, BLI was performed to detect the signal intensity changes at various developmental stages and at an interval of $<7$ days. The established Arc-Luc Tg HL mice exhibited clear and stable photon signals from the brain without interference during development. After surgical monocular deprivation during visual-critical period, large signal intensity changes in bioluminescence were observed in the mouse visual cortex. Exposure of mice to a novel object changed the photon distribution in the caudal and rostral cerebral areas. The temporal pattern of kainic-acid-induced Arc-Luc expression showed biphasic changes in signal intensity over $24 \mathrm{~h}$.
\end{abstract}

Conclusions: This study showed the advantages of using the mutant $\mathrm{HL}$ gene in BLI of Arc expression in the mouse brain at various developmental stages. Thus, the use of the Arc-LuC Tg HL mice enabled the tracking of neuronal-activity-dependent processes over a wide range from a focal area to the entire brain area with various time windows.

Keywords: Hairless, Arc, Development, Bioluminescence imaging

\section{Background}

Bioluminescence imaging (BLI) is a powerful research technique based on detection of light emission produced by oxidation of luciferin by luciferase $[1,2]$. The advantages of this technique in studies of small living animals are the following: high signal-to-noise $(\mathrm{S} / \mathrm{N})$ ratio with low background signal intensities, low limit and simplicity of detection, wide dynamic range of signal intensities, and applicability to genetic manipulation as well as continuous and quantitative analyses. BLI can also be

\footnotetext{
*Correspondence: hmori@med.u-toyama.ac.jp

1 Department of Molecular Neuroscience, Graduate School of Medicine and Pharmaceutical Sciences, University of Toyama, Toyama 930-0194, Japan

Full list of author information is available at the end of the article
}

applied repeatedly to the same animal, thus contributing to the reduction in the number of animals used. BLI can be applied to the detection of the biological signal intensity changes that affect the up- and down-regulations of transcription. Luciferase reporter animals are used in the research fields of inflammation [3], toxicology [4], oncology [5], angiogenesis [6], nutrition [7], and neurobiology [8]. Furthermore, split luciferase and bioluminescence resonance energy transfer (BRET) techniques have recently been developed for the detection of proteinprotein interaction $[9,10]$.

Previously, we established a novel reporter transgenic (Tg) mouse strain, namely, Arc-Luc Tg mice, in which the firefly luciferase gene $(L u c)$ is driven by the Arc promoter [11]. Arc, that is, the activity-regulated 
cytoskeleton-associated protein gene, is one of the immediate early genes (IEGs), which are highly responsive to various external stimuli in the central nervous system (CNS) [12, 13]. Using Arc-Luc Tg mice, we have successfully detected the bioluminescence signals of Arc-Luc in the adult mouse brain. The intensity of bioluminescence signals changed after physiological or pharmacological manipulations and the change well correlated with neuronal-activity-dependent Arc expression. Our Arc-Luc Tg mice were established using the C57BL/6 strain because this strain is one of the inbred strains most widely used in the fields of genetical engineering and neuroscience. However, its black fur, skin pigmentation, and hair regrowth after depilation interfere with BLI during development. Thus, the periods of BLI were restricted less than $24 \mathrm{~h}$ and at an interval of more than 4 weeks [11].

To extend the use of BLI at various mouse developmental stages, we have introduced a mutant HL gene into the original Arc-Luc Tg mice to overcome the abovementioned problems. Here, we examined the availability of $A r c-L u c$ Tg HL mice for BLI at various developmental stages under physiological and pathological conditions.

\section{Results}

\section{In vivo imaging of Arc-Luc Tg $\mathrm{HL}$ mice}

Previously, we successfully generated $A r c-L u c$ Tg mice for the BLI of Arc expression [11]. However, the genetic background of the Arc-Luc Tg mice is C57BL/6, and the black fur and skin pigmentation of this strain attenuate photon emission at various developmental stages (Fig. 1a). Thus, we introduced the mutant HL gene into the original Arc$L u c \mathrm{Tg}$ mice by crossing them with the HL mice. The established Arc-Luc Tg HL mice showed rapid induction of IEGs after kainic acid (KA) injection ( $A r c, p=0.026$; Egr-1, $p=0.023 ; c$-fos, $p=0.049$ ). The fold changes of these IEGs after KA injection in Arc-Luc Tg HL mice were the same as those in the C57BL/6 mouse strain (Fig. 1b, Additional file 1: Table S1, Arc, $p=0.16$; Egr$1, p=0.96$; $c$-fos, $p=0.20$ ). Using cooled CCD camera systems, we detected bioluminescence signals in areas around the nose, brain, paws, kidneys, and testes in the Arc-Luc Tg HL mice (Fig. 2a). The distribution of these signals was consistent with those previously reported for Arc expression and public database from transcriptome analysis [14]. The continuous BLI of an individual animal showed that the bioluminescence signal intensity in the brain gradually decreased from 4 to 8 weeks of age (Fig. 2a). Furthermore, we examined the expression levels of Luc and endogenous Arc proteins by western blot analysis and found that they were slightly higher in the cerebral cortex at 4 weeks of age than at 8 weeks of age (Fig. 2b, Additional file 1: Table S2). These developmental changes in protein expression levels correlated with

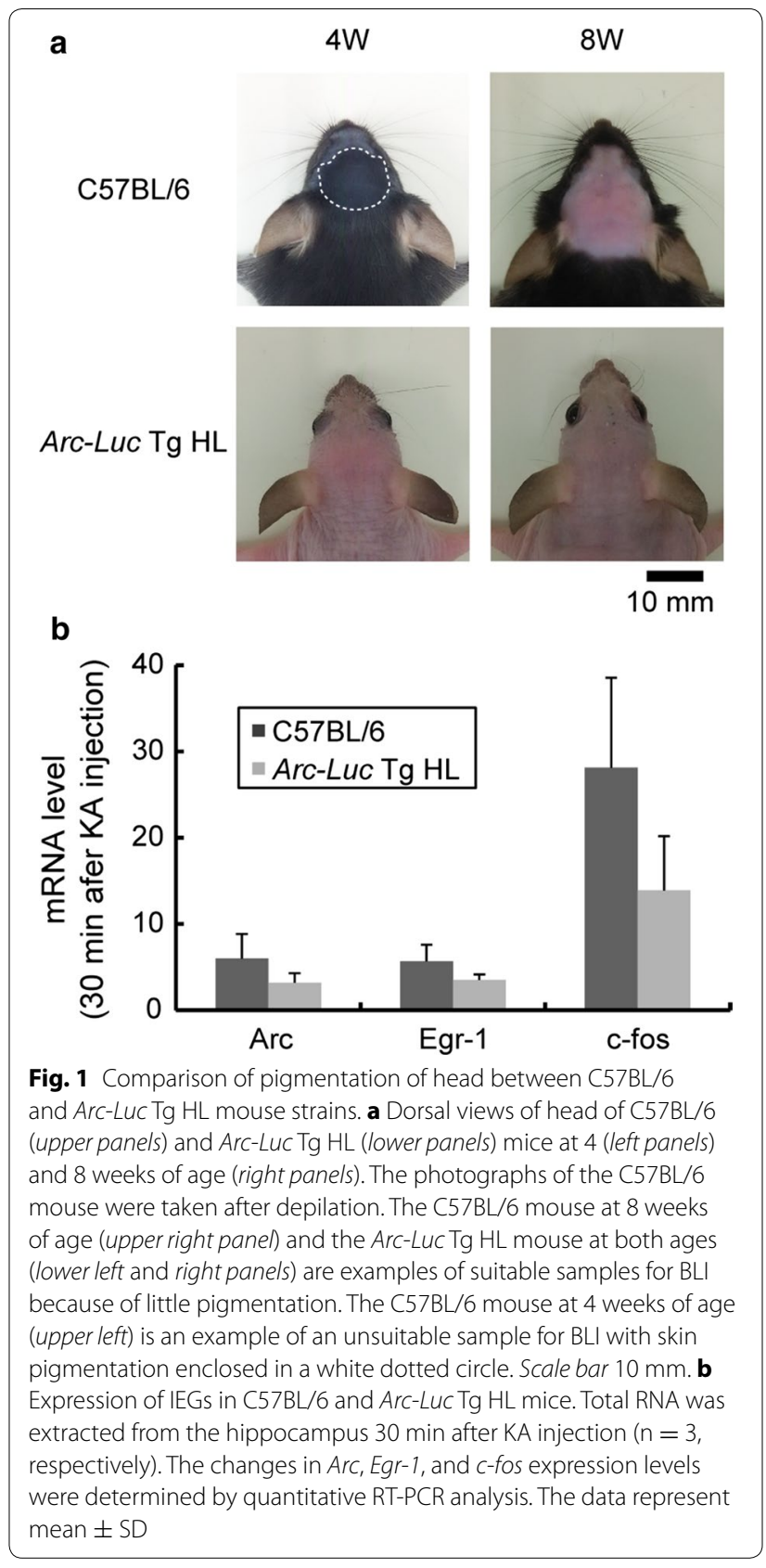

the changes in bioluminescence signal intensity. These findings demonstrate that changes in the Arc expression level in the entire body during mouse development can be noninvasively detected by the visualization of $A r c-L u c$ expression in Arc-Luc Tg HL mice.

\section{Monitoring responses to sensory stimuli}

$A r c$ is one of the IEGs, which are induced by various sensory stimuli. We previously reported the activitydependent and plastic changes in Arc expression in the visual cortex of adult Arc-Luc Tg mice [11]. In this 


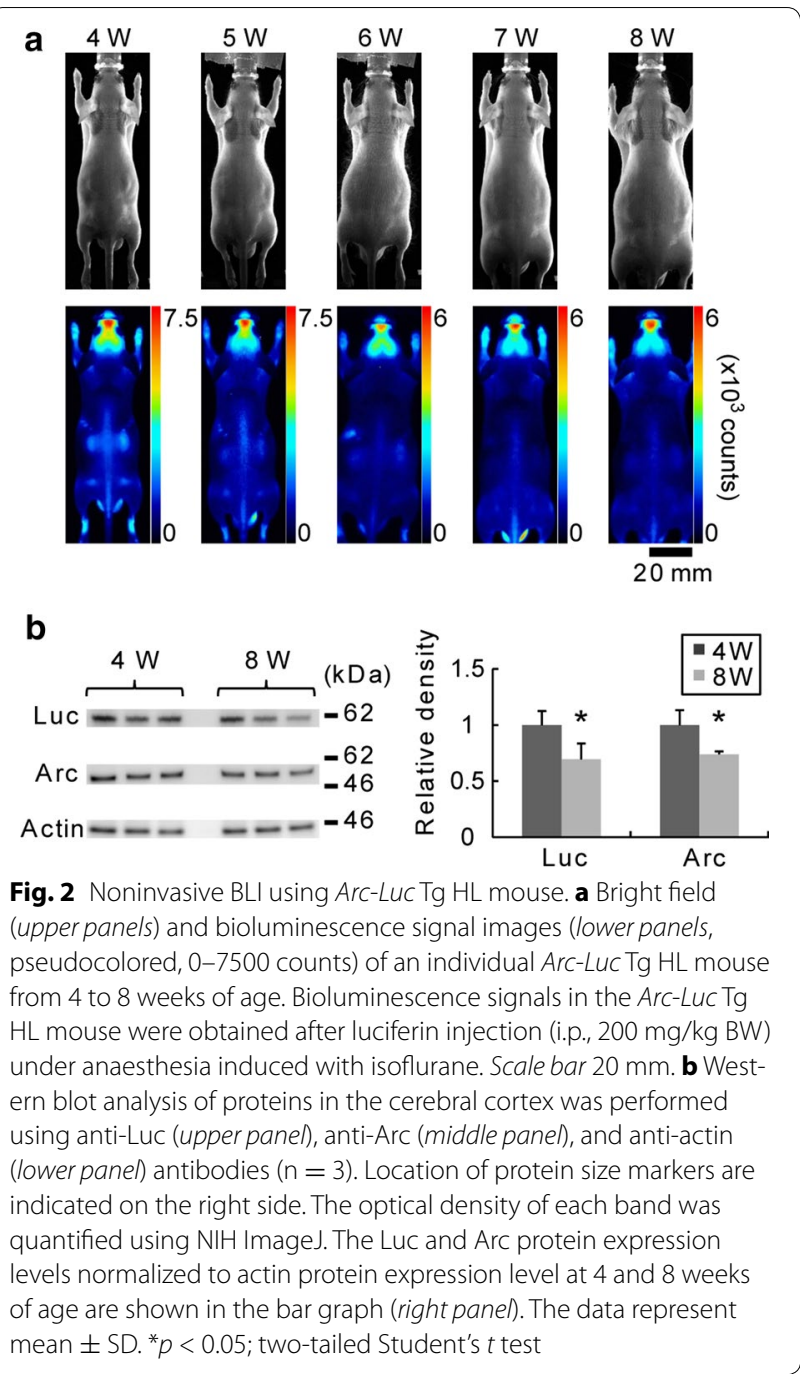

experiment, we extended the application of $A r c-L u c \mathrm{Tg}$ HL mice to continuous imaging at an interval of less than 7 days. We examined the pattern of changes in bioluminescence signal intensity during the development of mice in visual-critical period [Postnatal day 21 (P21)-P35] [15] with visual operation (Fig. 3a). The Arc-Luc Tg HL mice on P21 were monocularly deprived by eyelid suture. One day after monocular deprivation (MD1), the bioluminescence signal intensity significantly decreased in the brain region contralateral to the sutured eye after $3 \mathrm{~h}$ exposure to an intense light stimulus. Seven days after continuous monocular deprivation (MD7), the bioluminescence signal intensity in this region slightly increased (Fig. 3b). Furthermore, the reopening (RO) of the sutured eye followed by light exposure resulted in a marked increase in the bioluminescence signal intensity in this region (Fig. 3b). For the quantitative assessment of

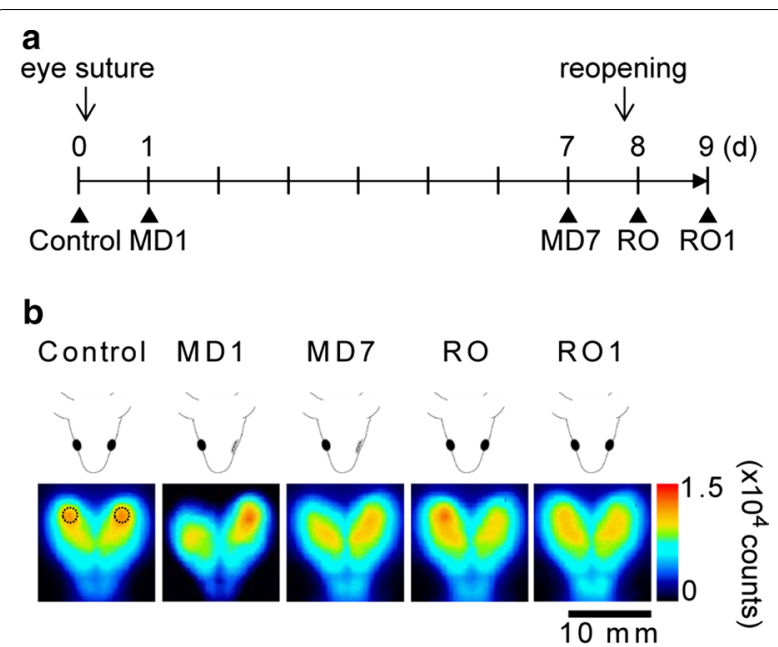

C

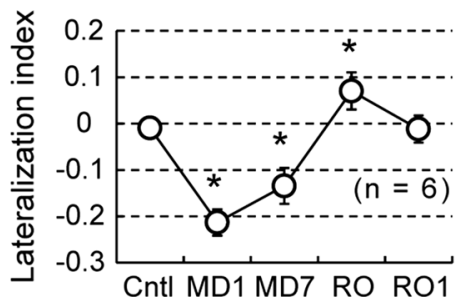

Fig. 3 Laterality of bioluminescence signals from visual cortex after monocular deprivation (MD) by suturing and reopening surgery. a Experimental schedule for monocular deprivation and reopening and BLI. The time points of BLI are indicated with black triangles. $\mathbf{b}$ The obtained bioluminescence images (pseudocolored, 0-15,000 counts) before eye suture (Control on P21), 1 day after MD (MD1 on P22), 7 days after MD (MD7 on P28), immediately after reopening (RO on P29), and 1 day after RO (RO1). Mice were exposed to an intense light for $3 \mathrm{~h}$ before each imaging time point. The locations of regions of interest (ROIs) are enclosed in dotted circles in the control image, and the obtained bioluminescence signal intensities are used for the calculation of lateralization index (LI) shown in (c). Scale bar $10 \mathrm{~mm}$. c Plots showing the LI under each condition shown in $(\mathbf{a})(n=6)$. $L$ I was calculated using the formula $(L-R) /(L+R)$, where $L$ and $R$ are the bioluminescence signal intensities of $R O I$ in the regions contralateral (left) and ipsilateral (right) to the sutured eye, respectively. LI was nearly zero under the control condition $(-0.009 \pm 0.004)$, decreased below zero under MD conditions (MD1, $-0.213 \pm 0.012 ;$ MD7, $-0.134 \pm 0.015)$, and increased immediately at $\mathrm{RO}(\mathrm{RO},+0.070 \pm 0.016)$, and finally returned close to the baseline at $\mathrm{RO} 1(\mathrm{RO} 1,-0.011 \pm 0.016)$. The data represent mean $\pm \mathrm{SD} .{ }^{*} p<0.05$; two-tailed Student's $t$ test

hemispheric dominance of Arc expression in the brain, we calculated lateralization index (LI), which is often used in functional magnetic resonance imaging (MRI) studies [16]. LI decreased to $-0.21( \pm 0.03)$ on MD1, and it slightly increased on MD7 but remained below zero $[-0.13( \pm 0.04)]$. Moreover, LI further increased to +0.07 $( \pm 0.04)$ after $\mathrm{RO}$ and returned to about 0 one day after RO (Fig. 3c, Additional file 1: Table S3). 
Spatial exploration in a novel environment induces Arc expression, and object and place recognition tasks induce the expression of some IEGs including Arc [17-19]. We carried out the evaluation of bioluminescence signal intensity changes associated with an object recognition (OR) task. Individually housed mice at 4 weeks of age were provided a novel plastic cube in their home cages (Fig. 4a). The object provided induced various exploratory behaviors of mice, such as approaching, sniffing, climbing, biting, and turning them over for several minutes. No statistically significant differences were detected in the total intensity of bioluminescence signals from the entire forebrain between the OR and control groups (Fig. $4 \mathrm{~b}, p=0.25$ ). However, we observed marked differences in the distribution of bioluminescence signals from the brain between the two groups. We divided ROIs in the forebrain into 28 rectangular blocks in the rostral to caudal direction (Fig. 4c). The distribution of relative bioluminescence signal intensity normalized to the signal intensity corresponding to the entire-forebrain photon density showed a down-regulation of the signals from the rostral region $(p<0.05$, at $0-1.4 \mathrm{~mm}$ from the rostral end) and the up-regulation of those from the posterior brain regions including the somatosensory, barrel, and visual cortices $(p<0.05,4.55-7.35 \mathrm{~mm}$ from the rostral end) (Fig. 4d, Additional file 1: Tables S4 and S5). Therefore, we concluded that the detection of bioluminescence signals in the Arc-Luc Tg HL mice clearly provided detailed neuronal-activity-dependent $A r c$ expression changes induced by sensory stimuli during development.

\section{Bioluminescence signal intensity changes after KA treatment}

KA is derived from a natural marine product from Digenea simplex [20] and is an excitatory amino acid receptor agonist selective to KA-type glutamate receptors. Acute treatment with KA is often carried out to produce a neurodegenerative disease model using laboratory animals and also induces Arc expression through synaptic activation. We reported the continuous increase in Arc-Luc expression level over $6 \mathrm{~h}$ after KA injection at the adult stage [11]. Here, we extend the examination of the temporal pattern of KA-induced Arc expression in the Arc-Luc $\mathrm{Tg}$ HL mice at 4 weeks of age (Fig. 5a). BLI was conducted after intraperitoneal KA injection (20 mg/kg BW) (Fig. 5b, c, Additional file 1: Table S6). The bioluminescence signal intensity increased three to fourfold within $3 \mathrm{~h}$ after $\mathrm{KA}$ injection, and then decreased to about twofold at $6 \mathrm{~h}$. Furthermore, a second increase was detected in the posterior region at $12 \mathrm{~h}$ and the intensity finally returned close to the baseline at $24 \mathrm{~h}$; this signal distribution pattern was different from that in the control saline-treated group. These results obtained from the Arc-Luc Tg HL mice suggest the advantages of temporal and quantitative

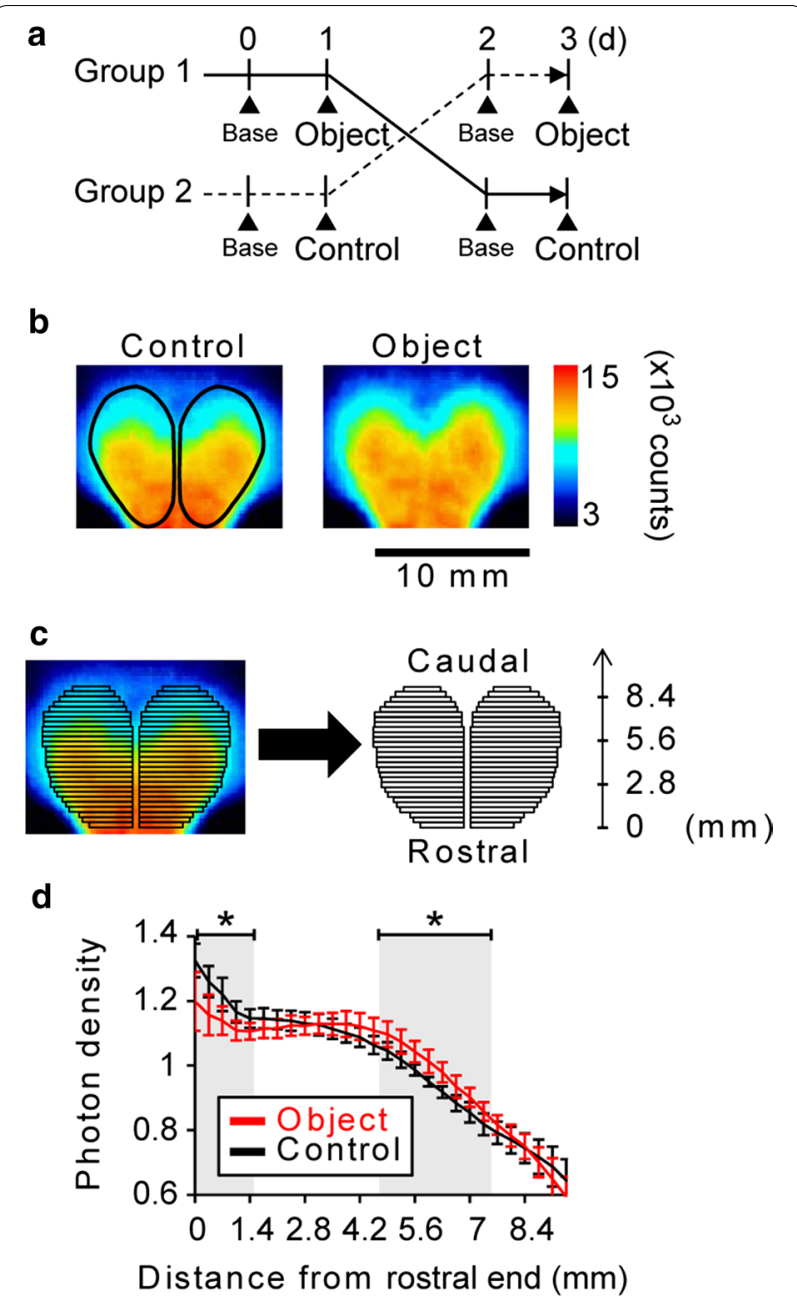

Fig. 4 Bioluminescence signal intensity changes associated with OR task. a Experimental schedule for OR task and BLI. Bioluminescence signal intensity was measured in each mouse $6 \mathrm{~h}$ after putting a plastic cube into their home cage (Object). For the Control, no object was presented. BLI was also performed without any treatment 1 day before the OR task (Base). The time points of BLI are indicated with black triangles. $\mathbf{b}$ Representative images of bioluminescence signals (pseudocolored, 3000-15,000 counts) in the Arc-Luc Tg HL mouse brain at 4 weeks of age for the Object and Control. Location of ROls in the forebrain is indicated in the Control image. Scale bar $10 \mathrm{~mm}$. c Subdivision of ROI in forebrain into smaller rectangles at $0.35 \mathrm{~mm}$ intervals in rostral to caudal direction. $\mathbf{d}$ Distribution of bioluminescence signals associated with $O R(n=7)$. Profiles of photon density throughout the forebrain for object presentation (red line) and control condition (black line). Each value obtained from ROls in (b) was normalized to the mean signal intensity (photon density $=1.0$ ) in the whole forebrain. Significant differences between the control and OR conditions are shown in gray $(0-1.4 \mathrm{~mm}$ and $4.55-7.35 \mathrm{~mm}$ from the rostral end) in the graph. The data represent mean \pm SD. ${ }^{*} p<0.05$; two-tailed Student's $t$ test

evaluations of the effect of pharmacological manipulation in vivo at various developmental stages without interferences from fur and skin pigmentation. 

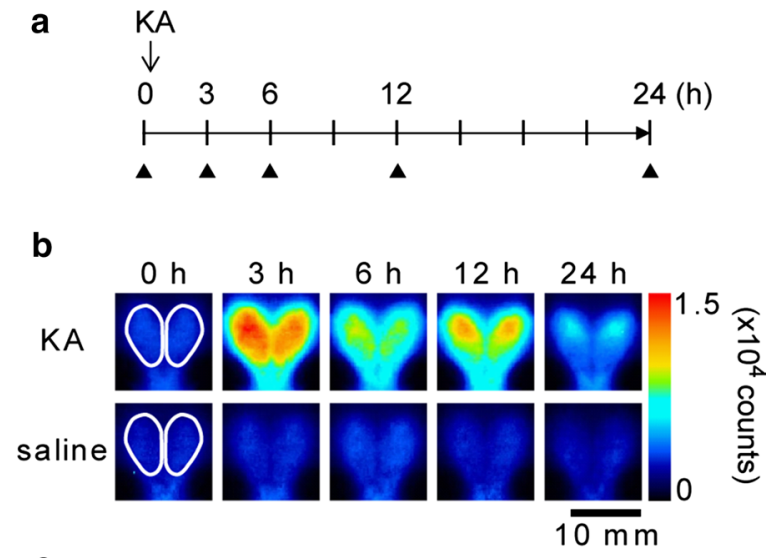

C

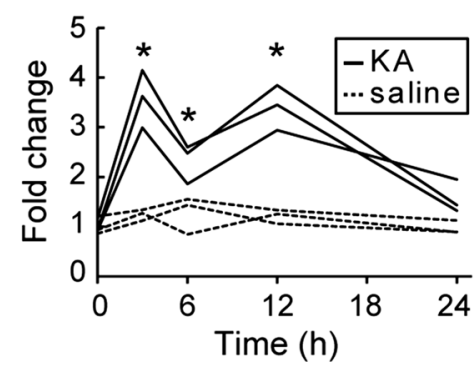

Fig. 5 Arc-Luc expression level changes after KA injection at 4 weeks of age. a Experimental schedule for BLI after KA injection. The time points of BLI are indicated with black triangles. b Images (pseudocolored, 0-15,000 counts) from representative mice taken at $0 \mathrm{~h}$ (before injection) and 3, 6, 12 and $24 \mathrm{~h}$ after KA (upper) or saline (lower) injection. The locations of ROls are indicated in the images at $0 \mathrm{~h}$. Scale bar, $10 \mathrm{~mm}$. c Changes in fold induction of bioluminescence signals within $24 \mathrm{~h}$ after KA injection $(n=3){ }^{*} p<0.05$; twotailed Student's $t$ test

\section{Discussion}

BLI is a noninvasive, simple, and quantitative method to evaluate brain function. However, the BLI of the mouse brain using the $\mathrm{C} 57 \mathrm{BL} / 6$ strain at various developmental stages has some problems such as light attenuation due to its black fur and skin pigmentation and the limited imaging period and interval caused by the hair regrowth after depilation. To overcome these problems, we established the $A r c-L u c$ Tg HL mouse strain for imaging neuronalactivity-dependent Arc expression at various developmental stages. We examined the reliability of this new mouse strain for investigating neuronal-activity-dependent $A r c$ expression processes.

The Arc-Luc Tg HL mice have several advantages that make them useful for investigating the neuronal-activitydependent changes during development. First, we can acquire stable bioluminescence signals from the brain without interferences after 3 weeks of age. Albino mice are also useful for BLI without shaving, but there remains the problem of light absorbance by white fur [21]. The application of our novel mouse strain to BLI is valuable in terms of the increase in the intensity of bioluminescence signals per specified time interval. As shown in Fig. 2, comparison of bioluminescence signal intensities at various developmental stages of the same animal enabled the detection of age-related changes in Arc expression level. Second, we can track bioluminescence signals daily and weekly. Because skin pigmentation occurs 5 days after depilation of the C57BL/6 mouse strain, we cannot measure bioluminescence signals in the original Arc-Luc Tg mice for more than five consecutive days. We have to wait at least 4 weeks for the full regrowth of hair for the next BLI. The Arc-Luc Tg HL mice on the other hand can be used daily and weekly to detect timedependent changes in Arc expression level in the brain by BLI (Figs. 2, 3, 4). Third, because the use of Arc-Luc Tg HL mice enables BLI for extended periods using the same animal, we can investigate the life-long effects of damage incurred from the early stages to the adulthood stage by BLI of Arc expression using fewer mice. However, despite these advantages of applying the Arc-Luc Tg HL mice to BLI, imaging at 1 or 2 weeks of age remains unfeasible because the hair loss in Arc-Luc Tg HL mice starts approximately on P14. There are several reports about the genes related to the regulation of hair growth and hair cycle [22-24], but the molecular mechanism underlying the initiation of first hair growth is as yet not fully understood. Further improvement of BLI to overcome this problem is necessary.

The Arc expression level in the brain slightly decreased from the early to adult stages (Fig. 2). Furthermore, we observed that the bioluminescence signal intensity of Arc-Luc in the submandibular gland increased with growth (data not shown). These findings suggest that Arc expression in mice was driven in age-dependent and tissue-specific manners.

In this study, BLI along with surgical monocular deprivation showed rapid changes in the bioluminescence signal intensity in the region contralateral to the deprived eye on MD1 and the subsequent recovery on MD7 (Fig. 3). Furthermore, continuous BLI showed the transient up-regulation of signals in the region contralateral to the deprived eye by reopening (LI shifted to over zero on the day of RO). These changes in bioluminescence signal intensity can be explained by reduced neuronal activity in contralateral visual cortex (MD1), recovery of neuronal activity in contralateral visual cortex by increase of the input from open eye (MD7), and increased neuronal activity in contralateral visual cortex by reopening of deprived eye (RO). Some reports indicate the changes in Arc expression during visual-critical period in the visual cortex $[25,26]$. Taken together with these previous reports, our results suggest that bioluminescence signal 
intensity changes caused by visual operation are induced by up- and down regulation of neuronal activity in visual cortex at a critical period.

Arc is induced by the OR task and spatial exploration in novel environments [17-19]. On the basis of this finding, we examined the signal intensity changes of Arc associated with the OR task. We successfully detected an increase in bioluminescence signal intensity in the posterior region of the brain including the somatosensory, barrel, and visual cortices of the mice after exposure to the novel object in their home cages (Fig. 4). Our findings suggest that a novel object is mainly processed using the senses of touch and sight. Although it is generally believed that mice mainly use the senses of smell and touch for OR, visual information also must be used for OR. Furthermore, the use of Arc-Luc Tg HL mice in combination with behavioral analysis may lead to the development of a technique for monitoring the neuronal activity in higher brain functions, such as recognition, emotion, and learning and memory.

In Fig. 5, we detected biphasic bioluminescence signal changes with a $9 \mathrm{~h}$ interval between the early ( $3 \mathrm{~h}$ ) and late phases (12 h) after KA injection in the Arc-Luc Tg HL mice. The rapid and protein-synthesis-independent transcription of $\operatorname{Arc}$ as an IEG is based on 'promoter proximal Pol II stalling' [27] and a specific enhancer with highly responsive to neuronal activity is located in the Arc promoter [28]. On the other hand, Arc expression under the regulation of new protein synthesis resulted in a late response [29, 30]. Actually, several studies showed a biphasic Arc induction with an 8-10 h interval and the necessity of protein synthesis for late induction of Arc after an electroconvulsive shock [31] and contextual fear conditioning [32]. Considering the regulation of $\mathrm{Arc}$ expression, the same molecular mechanism may explain our results. However, an increase in bioluminescence signal intensity after KA injection might be a result of greater entry of D-luciferin caused by the disruption of blood brain barrier. Further study is required for the evaluation of D-luciferin penetrance after status epilepticus.

\section{Conclusions}

We have shown the advantages of an application of the HL gene to BLI of the mouse brain in this study. Without interferences, the Arc-Luc Tg HL mice exhibited clear and stable photon signals and enabled the tracking of $\operatorname{Arc}$ expression under physiological and pathological conditions during development and at a short time interval. These results indicate that this mouse strain can be used for rapid and quantitative assessments of neuronal-activity-dependent processes in the mouse brain over a wide range from a focal area to the entire brain area with various time windows.

\section{Methods}

\section{Animals}

The experimental procedures used in this study were carried out in accordance with the Guidelines for the Care and Use of Laboratory Animals of the University of Toyama, and were approved by the Animal Experiment Committee of University of Toyama (Authorization No. A2011med-13). This study complies with the ARRIVE (Animal Research: Reporting In Vivo Experiments) guidelines. $A r c-L u c \mathrm{Tg}$ HL mice were established by crossing Arc-Luc Tg C57BL/6 mice [11] with a hairless mouse strain (Hos:HRM, Hoshino Laboratory Animals, Inc., Japan). All the animals were maintained under standard laboratory conditions (12-12 h/light-dark cycle with light on at 7:00 am; room temperature, $22 \pm 2{ }^{\circ} \mathrm{C}$ ) in the Laboratory Animal Resource Center of the University of Toyama. The Arc-Luc Tg HL mice were genotyped by PCR for the firefly luciferase gene ( $L u c 2$, Promega, Madison, WI, USA) and Southern blot analysis for Arc-Luc as previously described [11]. The heterozygous Arc-Luc Tg HL mice were bred and the resulting heterozygous and homozygous Arc-Luc Tg HL mice were used in this study.

\section{Quantitative RT-PCR analysis}

Total RNA was isolated from adult C57BL/6 and $A r c-$ Luc Tg HL mouse hippocampi 30 min after KA injection $[20 \mathrm{mg} / \mathrm{kg} \mathrm{BW}$, intraperitoneally (i.p.)] with TRIzol Reagent (Invitrogen, Carlsbad, CA, USA). Saline was injected as a control condition. Total RNA $(1 \mu \mathrm{g})$ was reverse-transcribed using ReverTra Ace (Toyobo, Tokyo, Japan) in accordance with the manufacturer's protocols. Quantitative PCR amplification was performed using the Mx3005P qPCR system (Agilent Technologies, Santa Clara, CA, USA) with Thunderbird SYBR qPCR Mix (Toyobo, Tokyo, Japan) for comparative quantification. The $\mathrm{Ct}$ of glyceraldehyde-3-phosphate dehydrogenase (GAPDH) was used for the calculation of the relative quantities of target genes. The following primers were used: 5'-GAGAGCTGAAAGGGTTGCAC-3' and 5'-GCCTT GATGGACTTCTTCCA-3' for Arc, $5^{\prime}$-TATACTGGCCG CTTCTCCCT-3' ${ }^{\prime}$ and $5^{\prime}$-AGAGGTCGGAGGATTGGT CA-3' for Egr-1, 5'-CTGAGATTGCCAATCTGCTG-3' and $5^{\prime}$-AGACATCTCCTCTGGGAAGC- $3^{\prime}$ for $c$-fos and 5'-ACAGTCCATGCCATCACTGC-3' and 5' ${ }^{\prime}$-TAGGAAC ACGGAAGGCCATG-3' for GAPDH.

\section{BLI of live mice}

In vivo BLI was performed as described previously [11]. Briefly, individually housed mice were anaesthetized by inhalation of isoflurane (1.5\% in air) and intraperitoneally (i.p.) injected with D-luciferin (AAT Bioquest, Sunnyvale, CA, USA) dissolved in phosphate-buffered saline (PBS, $\mathrm{pH} 7.4$ ) at $200 \mathrm{mg} / \mathrm{kg}$ body weight (BW). Ten minutes 
after luciferin injection, bioluminescence signal intensity was repeatedly measured for 30-120 s using an in vivo imaging system (Clairvivo OPT; Shimadzu Co., Kyoto, Japan) with $4 \times 4$ binning and without using an optical filter or excitation LED lights, as reported previously [11]. Bioluminescence signal intensity from captured images was calculated by region of interest (ROI) analysis using NIH ImageJ. Background bioluminescence signal intensity in non- $\mathrm{Tg}$ mice was subtracted from all values. Data were expressed as the mean number of photons per pixel (counts) in the ROI.

\section{Western blot analysis}

Deeply anaesthetized mice (sodium pentobarbital, $150 \mathrm{mg} / \mathrm{kg} \mathrm{BW}$, i.p. injected) were transcardially perfused with ice-cold PBS. The cerebral cortex was dissected and homogenized in ice-cold Mammalian Tissue Extraction Reagent (Thermo Scientific, Rochford, IL, USA) supplemented with $1 \mathrm{mM}$ phenylmethylsulfonyl fluoride and a protease inhibitor cocktail (Roche Diagnostics, Mannheim, Germany). The extracted proteins $(10 \mu \mathrm{g})$ separated by SDS-PAGE were transferred to polyvinylidene difluoride (PVDF) membranes (GE Healthcare, Little Chalfont, Buckinghamshire, UK). After blocking with 5\% skim milk in PBS containing 0.1\% Tween20, the membranes were incubated with the appropriate primary antibodies overnight at $4{ }^{\circ} \mathrm{C}$. The membranes were washed and incubated with the appropriate horseradish peroxidase (HRP)-conjugated secondary antibodies. Protein bands were visualized using the ECL Plus Western Blotting Detection Reagents (GE Healthcare, Little Chalfont, Buckinghamshire, UK) and measured using the ImageQuant LAS-4000 system (GE Healthcare, Little Chalfont, Buckinghamshire, UK).

The following antibodies were used: rabbit anti-Arc (1:1000, Synaptic Systems, Goettingen, Germany), goat anti-luciferase (1:1000, Promega, Fitchburg, WI, USA), rabbit anti-actin (1:2000 Santa Cruz Biotechnology, Dallas, TX, USA), HRP-conjugated goat anti-rabbit (1:2000, Bio-Rad, Hercules, CA, USA), and HRP-conjugated rabbit anti-goat (1:2000, Invitrogen, Camarillo, CA, USA) antibodies.

\section{Surgical monocular deprivation}

Mice were anaesthetized with sodium pentobarbital (50 mg/kg BW i.p. injected) and the eyelids were sutured using 6-0 vicryl after BLI on postnatal day 21 (P21). Monocular deprivation (MD) was continued for 7 days and checked for complete eye closure, and the sutured eye was reopened on P29. Animals with signs of injury or infection were excluded from this experiment. BLI was conducted after exposure to an intense light for $3 \mathrm{~h}$ at each time point (Fig. 3). LI [16] was calculated using the equation $\mathrm{LI}=(\mathrm{L}-\mathrm{R}) /(\mathrm{L}+\mathrm{R})$, where $\mathrm{L}$ and $\mathrm{R}$ are the signal intensities of the ROIs in the brain regions contralateral (left) and ipsilateral (right) to the deprived eye, respectively. A positive LI indicates deprived eye dominance, whereas a negative LI indicates nondeprived eye dominance.

\section{Exposure to novel object}

Individually caged mice at 4 weeks of age were used as subjects. The mice were divided into the object recognition (OR) and control groups. On the first day, bioluminescence signal intensity was measured in each mouse without any treatments. The next day, the mice in the OR group were exposed to a novel cube in their home cages for $6 \mathrm{~h}$ followed by BLI. The cube was a plastic Lego block. Mice in the control group were kept in their home cages until signal intensity measurement. Then, the mice in the OR and control groups were switched and treated as described above. Small rectangular ROIs $(2.5-5.3 \mathrm{~mm}$ width $\times 0.35 \mathrm{~mm}$ length) in the rostral to caudal direction were set on a template of the forebrain observed in the acquired photon image (Fig. 4b, c). The photon density in each rectangular ROI was calculated and normalized to the mean photon density in the entire forebrain.

\section{Exposure to $\mathrm{KA}$}

Mice at 4 weeks of age were separated into individual cages and i.p. injected with $\mathrm{KA}(20 \mathrm{mg} / \mathrm{kg} \mathrm{BW})$ dissolved in saline. KA-treated mice showed limbic motor seizure for 1-3 h. Control mice were injected with the same volume of saline. We measured bioluminescence signal intensity before (at $0 \mathrm{~h}$ ) and after $\mathrm{KA}$ injection (at 3, 6, 12 and $24 \mathrm{~h}$ ). The area enclosed in a white line corresponding to the entire brain (Fig. 5b) was used as a ROI. The bioluminescence signal changes in each group were expressed as a fold induction based on the signal intensity obtained at $0 \mathrm{~h}$.

\section{Statistical analysis}

All values are presented as mean \pm standard deviation (SD). The statistical significance of differences was determined by two-tailed Student's $t$ test. Values of $p<0.05$ were considered significant.

\section{Additional file}

Additional file 1: Table S1. IEGs mRNA levels relative to GAPDH at 30 min after KA injection in Fig. 1b. Table S2, Relative density of Luc and Arc proteins in Fig. 2b. Table S3, LI values under visual operation in Fig. 3c. Table S4, Photon density throughout the forebrain of Control in Fig. 4d. Table S5, Photon density throughout the forebrain of OR in Fig. 4d. Table S6, Fold induction of bioluminescence signals by KA in Fig. $5 \mathrm{c}$. 


\begin{abstract}
Abbreviations
Arc: activity-regulated cytoskeleton-associated protein gene; BLI:

bioluminescence imaging; BRET: bioluminescence resonance energy transfer BW: body weight; CNS: central nervous system; HL: hairless; IEGs: immediate early genes; i.p.: intraperitoneally; KA: kainic acid; LI: lateralization index; Luc: luciferase; MD: monocular deprivation; MRI: magnetic resonance imaging; OR: object recognition; PBS: phosphate-buffered saline; PVDF: polyvinylidene difluoride; RO: reopening; ROI: region of interest; SD: standard deviation; S/N: signal-to-noise; Tg: transgenic.
\end{abstract}

\section{Authors' contributions}

$\mathrm{HI}, \mathrm{Tl}$, and $\mathrm{HM}$ designed this study. $\mathrm{HI}$ and $\mathrm{HY}$ contributed to the establishment and maintenance of the transgenic animals. HI performed all animal surgery and in vivo imaging. $\mathrm{HI}$ and $\mathrm{HM}$ performed the data analysis. $\mathrm{HI}, \mathrm{Tl}$ and $\mathrm{HM}$ wrote the paper. All authors read and approved the final manuscript.

\section{Author details}

${ }^{1}$ Department of Molecular Neuroscience, Graduate School of Medicine and Pharmaceutical Sciences, University of Toyama, Toyama 930-0194, Japan. 2 Division of Animal Resources and Development, Life Science Research Center, University of Toyama, Toyama 930-0194, Japan.

\section{Acknowledgements}

This research was supported by grants from the Food Safety Commission of Japan (No. 1001) and Scientific Research on Innovation Area (Comprehensive Brain Science Network, No. 221S003) from the Ministry of Education, Science, Sports and Culture of Japan.

\section{Competing interests}

The authors declare that they have no competing interests.

\section{Availability of data and materials}

The datasets supporting the conclusions of this study are included within the article and are also provided as an additional file. The Arc-Luc Tg HL mice will be available to the research community.

\section{Ethical approval}

The experimental procedures used in this study were carried out in accordance with the Guidelines for the Care and Use of Laboratory Animals of the University of Toyama, and were approved by the Animal Experiment Committee of University of Toyama (Authorization No. A2011 med-13). This study complies with the ARRIVE (Animal Research: Reporting In Vivo Experiments) guidelines.

Received: 18 June 2016 Accepted: 12 January 2017

Published online: 23 January 2017

\section{References}

1. Sato A, Klaunberg B, Tolwani R. In vivo bioluminescence imaging. Comp Med. 2004;54:631-4.

2. Zinn KR, Chaudhuri TR, Szafran AA, O'Quinn D, Weaver C, Dugger K, et al. Noninvasive bioluminescence imaging in small animals. ILAR J. 2008;49:103-15.

3. Zhang N, Ahsan MH, Purchio AF, West DB. Serum amyloid A-luciferase transgenic mice: response to sepsis, acute arthritis, and contact hypersensitivity and the effects of proteasome inhibition. J Immunol. 2005;174:8125-34.

4. Ciana P, Raviscioni M, Mussi P, Vegeto E, Que I, Parker MG, et al. In vivo imaging of transcriptionally active estrogen receptors. Nat Med. 2003;9:82-6.

5. Vooijs M, Jonkers J, Lyons S, Berns A. Noninvasive Imaging of Spontaneous Retinoblastoma Pathway-dependent Tumors in Mice. Cancer Res. 2002;62:1862-7.

6. Greene JM, Dunaway CW, Bowers SD, Rude BJ, Feugang JM, Ryan PL. In vivo monitoring of fetoplacental Vegfr2 gene activity in a murine pregnancy model using a Vegfr2-luc reporter gene and bioluminescent imaging. Reprod Biol Endocrinol. 2011;9:51.
7. Austenaa LMI, Carlsen H, Ertesvag A, Alexander G, Blomhoff HK, Blomhoff R. Vitamin A status significantly alters nuclear factor-kappaB activity assessed by in vivo imaging. FASEB J. 2004;18:1255-7.

8. Zhu L, Ramboz S, Hewitt D, Boring L, Grass DS, Purchio AF. Non-invasive imaging of GFAP expression after neuronal damage in mice. Neurosci Lett. 2004;367:210-2.

9. Audet M, Lagacé M, Silversides DW, Bouvier M. Protein-protein interactions monitored in cells from transgenic mice using bioluminescence resonance energy transfer. FASEB J. 2010;24:2829-38.

10. Ozawa T, Kaihara A, Sato M, Tachihara K, Umezawa Y. Split luciferase as an optical probe for detecting protein-protein interactions in mammalian cells based on protein splicing. Anal Chem. 2001;73:2516-21.

11. Izumi $H$, Ishimoto $T$, Yamamoto $H$, Nishijo $H$, Mori $H$. Bioluminescence imaging of Arc expression enables detection of activity-dependent and plastic changes in the visual cortex of adult mice. Brain Struct Funct. 2011;216:91-104.

12. Korb E, Finkbeiner S. Arc in synaptic plasticity: from gene to behavior. Trends Neurosci. 2011;34:591-8.

13. Okuno $H$. Regulation and function of immediate-early genes in the brain: beyond neuronal activity markers. Neurosci Res. 2011;69:175-86.

14. Lizio M, Harshbarger J, Shimoji H, Severin J, Kasukawa T, Sahin S, et al. Gateways to the FANTOM5 promoter level mammalian expression atlas. Genome Biol. 2015;16:22

15. Espinosa JS, Stryker MP. Development and plasticity of the primary visual cortex. Neuron. 2012;75:230-49.

16. Seghier ML. Laterality index in functional MRI: methodological issues. Magn Reson Imaging. 2008;26:594-601.

17. Montag-Sallaz M, Montag D. Learning-Induced arg 3.1/arc mRNA Expression in the Mouse Brain. Learn Mem. 2003;10:99-107.

18. Soulé J, Penke Z, Kanhema T, Alme MN, Laroche S, Bramham CR, et al. Object-place recognition learning triggers rapid induction of plasticityrelated immediate early genes and synaptic proteins in the rat dentate gyrus. Neural Plast. 2008;2008:269097.

19. Ramírez-Amaya V, Vazdarjanova A, Mikhael D, Rosi S, Worley PF, Barnes CA. Spatial exploration-induced Arc mRNA and protein expression: evidence for selective, network-specific reactivation. J Neurosci. 2005;25:1761-8.

20. Takemoto T. Isolation and structural identification of naturally occurring excitatory amino acids. In: McGeer EG, Olney JW, McGeer PL, editors. Kainic acid as a tool in neurobiology. New York: Raven Press; 1978. p. 1-15.

21. Wu JC, Sundaresan G, lyer M, Gambhir SS. Noninvasive optical imaging of firefly luciferase reporter gene expression in skeletal muscles of living mice. Mol Ther. 2001;4:297-306.

22. Beaudoin GMJ, Sisk JM, Coulombe PA, Thompson CC. Hairless triggers reactivation of hair growth by promoting Wnt signaling. Proc Natl Acad Sci USA. 2005;102:14653-8.

23. Kiso M, Tanaka S, Saba R, Matsuda S, Shimizu A, Ohyama M, et al. The disruption of Sox21-mediated hair shaft cuticle differentiation causes cyclic alopecia in mice. Proc Natl Acad Sci USA. 2009;106:9292-7.

24. Skorija K, Cox M, Sisk JM, Dowd DR, MacDonald PN, Thompson CC, et al. Ligand-independent actions of the vitamin $D$ receptor maintain hair follicle homeostasis. Mol Endocrinol. 2005;19:855-62.

25. Gao M, Sossa K, Song L, Errington L, Cummings L, Hwang H, et al. A specific requirement of Arc/Arg3.1 for visual experience-induced homeostatic synaptic plasticity in mouse primary visual cortex. J Neurosci. 2010;30:7168-78.

26. Tagawa Y, Kanold PO, Majdan M, Shatz CJ. Multiple periods of functional ocular dominance plasticity in mouse visual cortex. Nat Neurosci. 2005;8:380-8.

27. Saha RN, Wissink EM, Bailey ER, Zhao M, Fargo DC, Hwang J-Y, et al. Rapid activity-induced transcription of Arc and other IEGs relies on poised RNA polymerase II. Nat Neurosci. 2011;14:848-56.

28. Kawashima T, Okuno H, Nonaka M, Adachi-Morishima A, Kyo N, Okamura $M$, et al. Synaptic activity-responsive element in the Arc/Arg3.1 promoter essential for synapse-to-nucleus signaling in activated neurons. Proc Natl Acad Sci USA. 2009;106:316-21.

29. Fukuchi M, Nakashima F, Tabuchi A, Shimotori M, Tatsumi S, Okuno H, et al. Class I histone deacetylase-mediated repression of the proximal promoter of the activity-regulated cytoskeleton-associated protein gene regulates its response to brain-derived neurotrophic factor. J Biol Chem. 2015:290:6825-36. 
30. Li L, Carter J, Gao X, Whitehead J, Tourtellotte WG. The neuroplasticityassociated arc gene is a direct transcriptional target of early growth response (Egr) transcription factors. Mol Cell Biol. 2005;25:10286-300.

31. Penke Z, Chagneau C, Laroche S. Contribution of Egr1/zif268 to activitydependent Arc/Arg3.1 transcription in the dentate Gyrus and area CA1 of the hippocampus. Front Behav Neurosci. 2011;5:48.
32. Nakayama D, Iwata H, Teshirogi C, Ikegaya Y, Matsuki N, Nomura H. Long-delayed expression of the immediate early gene Arc/Arg3.1 refines neuronal circuits to perpetuate fear memory. J Neurosci. 2015;35:819-30.

\section{Submit your next manuscript to BioMed Central and we will help you at every step:}

- We accept pre-submission inquiries

- Our selector tool helps you to find the most relevant journal

- We provide round the clock customer support

- Convenient online submission

- Thorough peer review

- Inclusion in PubMed and all major indexing services

- Maximum visibility for your research

Submit your manuscript at

www.biomedcentral.com/submit 